\title{
Statins reduce melanoma development and metastasis through MICA overexpression
}

\author{
Christine Pich ${ }^{1,2,3}$, lotefa Teiti 1,2,3, Philippe Rochaix ${ }^{2}$, Bernard Mariamé ${ }^{3,4}$, Bettina Couderc ${ }^{2,3,5}$, Gilles Favre ${ }^{1,2,3}$ \\ and Anne-Françoise Tilkin-Mariamé 1,2,3* $^{2}$
}

\author{
1 INSERM/UPS UMR 1037, Cancer Research Center of Toulouse, Toulouse, France \\ 2 Institut Claudius Regaud, Toulouse, France \\ ${ }^{3}$ Université Paul Sabatier, Toulouse, France \\ 4 INSERM/UPS UMR 1043, Physiopathology Center of Toulouse-Purpan, Toulouse, France \\ ${ }^{5}$ EA 4553, University Cancer Clinic, Toulouse, France
}

\section{Edited by:}

Fabrizio Mattei, Istituto Superiore di Sanità, Italy

\section{Reviewed by:}

Geraldine O. Canny, Geneva

Foundation for Medical Education and Research, Switzerland

Yang Bian, The Research Institute at Nationwide Children's Hospital, USA

*Correspondence:

Anne-Françoise Tilkin-Mariamé, INSERM UMR 1037, Cancer Research Center of Toulouse, Institut Claudius Regaud, 20-24, Rue du Pont Saint Pierre, Toulouse 31052, France. e-mail: tilkin.anne@claudiusregaud.fr
Survival of melanoma patients after metastases detection remains short. Several clinical trials have shown moderate efficiency in improving patient survival, and the search for pharmacological agents to enhance the immune response and reduce melanoma metastases is still necessary. Statins block the mevalonate pathway, which leads to decreases in GTPase isoprenylation and activity, particularly those of the Ras superfamily. They are widely used as hypocholesterolemic agents in cardiovascular diseases and several studies have shown that they also have protective effects against cancers. Furthermore, we have previously demonstrated that treatment of melanoma cells with inhibitors of the mevalonate pathway, such as statins, favor the development of specific adaptive immune responses against these tumors. In the present study, we tested statin impact on the innate immune response against human metastatic melanoma cells. Our data shows that treatment of two human melanoma cell lines with statins induced a weak but significant increase of $\mathrm{MHC}$ class I Chain-related protein A (MICA) membrane expression. Peroxisome Proliferator-Activated Receptor gamma is involved in this statin-induced MICA overexpression, which is independent of Ras and Rho GTPase signaling pathways. Interestingly, this MICA overexpression makes melanoma cells more sensitive to in vitro lysis by NK cells. The impact of statin treatment on in vivo development of melanoma tumors and metastases was investigated in nude mice, because murine NK cells, which express NKG2D receptors, are able to recognize and kill human tumor cells expressing MICA. The results demonstrated that both local tumor growth and pulmonary metastases were strongly inhibited in nude mice injected with statin-treated melanoma cells. These results suggest that statins could be effective in melanoma immunotherapy treatments.

Keywords: statins, melanoma, MICA, NK cells

\section{INTRODUCTION}

Cutaneous melanomas are the most aggressive skin cancers. They arise from melanocytes and, after horizontal and vertical growth, extravasate into the draining lymph nodes and blood vessels, from which they can metastasize. Patient survival following metastatic detection is usually short. Therefore, current treatments target metastatic growth. Inhibitors of BRAF mutated V600E were validated by several clinical trials but their efficiency is not long lasting and is mostly restricted to BRAF V600E mutated tumors (Flaherty et al., 2010; Weeraratna, 2012). Other treatments with antibodies directed against the CTLA- 4 molecule have shown efficiency in metastatic melanoma (Hodi et al., 2010), but these antibodies can also induce deleterious inflammation (Berman et al., 2010). Thus, the search for pharmacological agents to enhance the immune response to reduce melanoma metastases is still necessary.

Statins are used by millions of people as hypocholesterolemic agents in cardiovascular and cerebrovascular diseases. Additionally, retrospective studies have shown that, besides this hypocholesterolemic activity, statins can also have a protective effect against the development of cancers, particularly melanomas (Boudreau et al., 2010; Jacobs et al., 2011). Statins are HMGCoA reductase inhibitors, which block the mevalonate synthesis pathway. By inhibiting this metabolic pathway, statins also inhibit the activity of numerous GTPases of the Ras superfamily as they require the isoprenoid chains, synthesized from the mevalonate, for their function and localization (Rikitake and Hirata, 2009). Statins also have pleiotropic effects, including the enhancement of Peroxisome Proliferator-Activated Receptor gamma (PPAR $\gamma$ ) activities (Balakumar and Mahadevan, 2012). PPAR $\gamma$ is a transcription factor that is overexpressed in several types of cancer (Peters et al., 2012), particularly melanomas (Meyer et al., 2009).

We have previously shown that treatments of melanoma cells with mevalonate pathway pharmacological inhibitors, notably statins, and in combination with IFN $\gamma$ favor the anti-tumor adaptive immune response by inducing MHC class I and costimulatory molecule (CD80/CD86) overexpression (Tilkin-Mariame et al., 
2005; Sarrabayrouse et al., 2010). We then asked the question whether or not these inhibitors could also favor the anti-melanoma innate immune response. We were particularly interested in NK cells, which are among the most efficient effectors of the innate immune system and also recognize several molecules expressed on the surface of both stressed and tumor cells. A critical receptor expressed on NK cells is the NKG2D activating receptor, which is able to recognize tumor cells from murine or human origin (Cerwenka and Lanier, 2001; Fuertes et al., 2008). On tumor or stressed cells, this type II transmembrane receptor recognizes ligands, such as UL-Binding Proteins (ULBPs) and MHC class I Chain-related protein A (MICA) and B (MICB), all related to the MHC class I molecules (Lanier, 2008). NKG2D expression is essential for NK cell control of tumor development, as illustrated by NKG2D$\mathrm{KO}$ mice, which develop numerous tumors and die prematurely (Guerra et al., 2008).

In the present study, we analyzed statins' ability to regulate MICA expression in human melanoma cells and the consequences of this treatment on melanoma development. We demonstrated that treatment of two human melanoma cells with atorvastatin or lovastatin induced a weak but reproducible MICA membrane overexpression, provoking an increase of melanoma cell sensitivity to NK cell lysis in vitro. Moreover, we observed in vivo that atorvastatin pretreatment of these melanoma cells strongly reduced both local melanoma growth following subcutaneous injection as well as pulmonary metastases implantation after intravenous injections. This data suggests that statins could be interesting pharmacological inhibitors for melanoma immunotherapy as they favor the innate immune response against tumor cells.

\section{RESULTS}

\section{STATIN TREATMENT INDUCES MICA OVEREXPRESSION AND INCREASES MELANOMA SENSITIVITY TO NK-KILLING}

We tested whether treatment of human melanoma cells with statins could render these cells more immunogenic and more sensitive to NK cell destruction. LB1319-MEL human melanoma cells were treated for $48 \mathrm{~h}$ with $5 \mu \mathrm{M}$ atorvastatin. This treatment induced a 2.2-fold total MICA protein enhancement (Figure 1A) and also weakly enhanced MICA membrane expression (Figures 1B,C), but not the deleterious cleavage of membrane expressed MICA (Figure 1D). The $5 \mu \mathrm{M}$ dose of atorvastatin for $48 \mathrm{~h}$ was chosen after dose-response experiments as illustrated in Figure A1A in Appendix. This atorvastatin treatment is not toxic to LB1319-MEL cells, as their in vitro proliferation rate following treatment is still similar to the control (Figure 1E). Similar results were obtained with another human melanoma cell line, BB74MEL, which was treated with atorvastatin at 5 or $10 \mu \mathrm{M}$. Similar to LB1319-MEL cells, the BB74-MEL cells also exhibited weak membrane MICA overexpression in response to statins (Figure A1B in Appendix) without increasing the cleavage of membrane expressed MICA (Figure A1C in Appendix) or toxicity (Figure A1D in Appendix).

Recent studies showed that the increase of MICA and MICB expression on target tumor cells induced an increase in sensitivity to lysis by NK cells (Zhang et al., 2009; Chavez-Blanco et al., 2011). We therefore tested whether or not atorvastatin plays a role in increasing the sensitivity of melanoma cells to NK cell-mediated death. We isolated fresh NK cells from C57BL/6 splenocytes using magnetic cell separation (Miltenyi Biotec) and cocultivated them for $1 \mathrm{~h}$ with LB1319-MEL target cells either untreated or pretreated with atorvastatin. FACS analysis of tumor cell lysis showed that atorvastatin treatment induced a twofold increase in LB1319-MEL cell death as compared to untreated target cells (Figure 1F). In this experiment, corresponding to previously shown data, murine NK cells were able to recognize and kill human target cells by interaction of their NKG2D receptors with specific ligands expressed on human target cells, such as MICA, MICB, and ULBP (Cerwenka and Lanier, 2001; Fuertes et al., 2008).

To test whether another statin could also induce MICA membrane overexpression, LB1319-MEL cells were treated with $1 \mu \mathrm{M}$ lovastatin for $48 \mathrm{~h}$ and MICA expression was analyzed by flow cytometry. The $1 \mu \mathrm{M}$ dose of lovastatin was chosen after doseresponse experiments as illustrated in Figure A1E in Appendix. Similarly to atorvastatin, lovastatin induced a weak MICA membrane overexpression (Figure 1G).

Altogether, this data shows that statin treatment of human melanoma cells enhanced MICA protein and membrane expression and that these treatments were not toxic, but increased the sensitivity of the tumor cells to NK-induced cell death in vitro.

\section{ATORVASTATIN TREATMENT OF LB1319-MEL CELLS SLOWS TUMOR GROWTH AND REDUCES METASTATIC PULMONARY IMPLANTATION}

Based on our in vitro conclusions, we wondered if atorvastatin treatment of melanoma cells could increase sensitivity to the anti-tumor innate immune response in a mouse model. In summary, we performed subcutaneous injections of LB1319-MEL cells, either untreated or pretreated with $5 \mu \mathrm{M}$ atorvastatin for $48 \mathrm{~h}$, into the flank of nude mice. We were not able to perform this assay with BB74-MEL cells, as they do not grow in the nude mice. The resulting tumors showed that LB1319-MEL cells pretreated with atorvastatin grew drastically $(p<0.005)$ slower than untreated cells (Figure 2A). Interestingly, two mice among the six injected with pretreated LB1319-MEL cells rejected their tumor (Figure 2C), whereas no rejection was observed among the mice injected with untreated LB1319-MEL cells (Figure 2B). These results are consistent with the statin-induced MICA overexpression and enhancement of in vitro tumor cell death by NK cells. Furthermore, we used two other human melanoma cell lines: LB2033-MEL and LB583-MEL. These cells are MICA negative and remained negative following atorvastatin treatment as illustrated in Figure A2A in Appendix. We injected subcutaneously these melanoma cells either untreated or pretreated, as LB1319MEL, with $5 \mu \mathrm{M}$ atorvastatin. The resulting tumors showed that negative MICA cells both pretreated and untreated grew similarly (Figures A2B,C in Appendix). These results confirmed the involvement of statin-induced MICA overexpression in the tumor growth.

We also tested the effect of atorvastatin treatment on the in vitro migration of the LB1319-MEL cells. We did not observed any significant difference between the migration of treated and untreated cells (Figures A2D,E in Appendix). This data suggested that the atorvastatin-induced slow-down of in vivo tumor growth is not linked to an inhibition of tumor cells migration. 


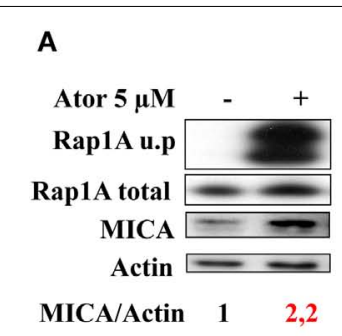

E

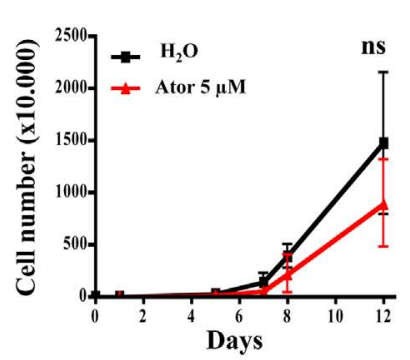

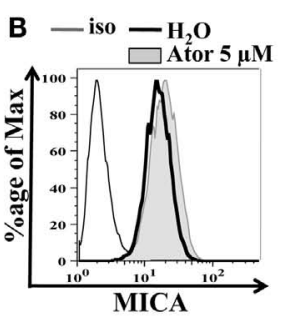

$\mathbf{F}$

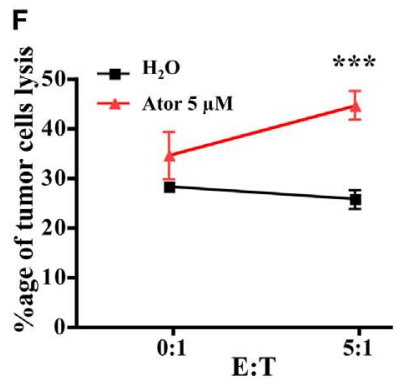

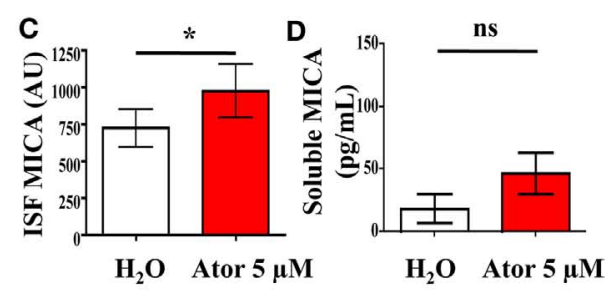

G -iso - EtOH

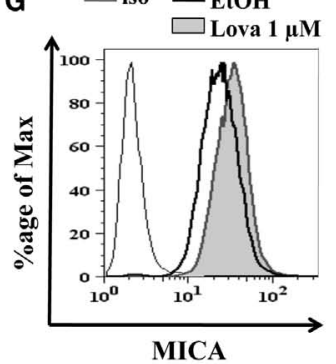

FIGURE 1 | Statin treatment induces MICA overexpression and increases NK-dependent cytotoxicity. LB1319-MEL cells were treated with $5 \mu \mathrm{M}$ atorvastatin for $48 \mathrm{~h}$ (Ator) or untreated $\left(\mathrm{H}_{2} \mathrm{O}\right)$. The atorvastatin treatment efficiency was controlled by the analysis of Rap1A unprenylation (Rap1A u.p) compared to the total protein (Rap1A total) (A). MICA total expression was analyzed by western blot (A), membrane expression by flow cytometry (B,C), and cleavage by ELISA (D) ( $n=3)$. To evaluate membrane antigen expression, the index of specific fluorescence (ISF) was calculated as indicated in the Section "Materials and Methods" (C). Results are expressed as mean $\pm \mathrm{SD}$ [error bars, $n=5$ experiments (C) or 3 experiments (D)]. ${ }^{*} P<0.5$; versus control condition using the Student $t$-test. Every 2-3 days pretreated (Ator) and untreated $\left(\mathrm{H}_{2} \mathrm{O}\right)$ LB1319-MEL cells were counted to evaluate the in vitro proliferation (E). ns; versus control condition using the two-way ANOVA test. Percentage of lysed LB1319-MEL cells pretreated (Ator) or untreated $\left(\mathrm{H}_{2} \mathrm{O}\right)$ after coculture with murine NK cells was evaluated with Cytoxilux kit

(Onco-immumin, Interchim). Murine NK cells were first isolated from three C57BL/6 spleens with CD49b MicroBeads (Miltenyi Biotec) (F). ${ }^{* *} P<0.001$; versus control condition using the two-way ANOVA test. LB1319-MEL cells were treated with $1 \mu \mathrm{M}$ lovastatin for $48 \mathrm{~h}$, and MICA membrane expression was analyzed by flow cytometry (G).
As NK cells play an important role in the control of metastatic processes (Mehlen and Puisieux, 2006), we assessed whether statin-induced MICA overexpression and subsequent sensitivity to the innate immune response could reduce the metastatic processes, more specifically the final step of pulmonary implantation. LB1319-MEL cells either untreated or pretreated with $5 \mu \mathrm{M}$ atorvastatin were injected intravenously into the tail vein of nude mice and the animals were sacrificed 16 days later. Thirty hours before sacrifice, the tumor and neoangiogenesis sensor IntegriSense was injected intravenously (Kimura et al., 2009; Mery et al., 2011; He et al., 2012; Valdivia et al., 2012). This fluorescent reagent allowed visualization and quantification of tumor implantations and neoangiogenesis in the lungs (Figures 2D,E). We observed that untreated LB1319-MEL cells colonized the lungs and induced a considerable neoangiogenesis, while atorvastatin-treated LB1319-MEL cells were less invasive with strongly decreased neoangiogenesis.

These results showed that statin pretreatment of melanoma cells reduced in vivo tumor development and metastasis.

\section{INHIBITION OF GTPases OF Ras SUPERFAMILY DOES NOT INDUCE MICA OVEREXPRESSION}

Proteins of the Ras superfamily play regulatory roles on essential cellular functions (Heasman and Ridley, 2008). Their activity can be blocked by statin treatment, because statins block mevalonate production by HMG-CoA reductase and consequently inhibit the production of isoprenyl PyroPhosphates (PP), geranyl PP, and farnesyl PP, which are the precursor molecules necessary to produce isoprenoid chains. These isoprenoid chains bind to the carboxy-terminal end of Ras superfamily proteins and allow adequate cellular localization and activity. To explain why statin treatment induced MICA overexpression, we hypothesized that statin could block a negative regulator of MICA expression. These negative regulatory proteins, blocked by statin treatment, could be isoprenylated proteins of the Ras superfamily.

We first tested the involvement of proteins of the Rho GTPases subfamily. The three highly related Rho isoforms, RhoA, B, and $\mathrm{C}$ share some effector proteins, but also show clear functional differences (Heasman and Ridley, 2008). To determine whether these Rho GTPases are involved in the regulation of MICA membrane expression, we used a bacterial inhibitor of RhoA, B, and $\mathrm{C}$ (Tat-C3 exoenzyme), which inhibits the Rho proteins activity by inducing ADP-ribosylation of these three GTPases. The results showed that treatment of LB1319-MEL cells with Tat-C3 actually induced downregulation of MICA rather than the expected increase (Figures 3A,B). Therefore, these Rho proteins effectively regulate MICA expression but in a positive way. We then used a siRNA strategy that allows the specific inhibition of RhoA, RhoB, or RhoC, to test whether one of them could behave differently and negatively regulate MICA expression. We first verified that these siRNAs were efficient in reducing RhoA, RhoB, or RhoC protein expression (Figures A3A-C in Appendix) and then tested their impact on MICA expression. Transfection of two RhoA-specific siRNAs in LB1319-MEL cells did not induce any modification 


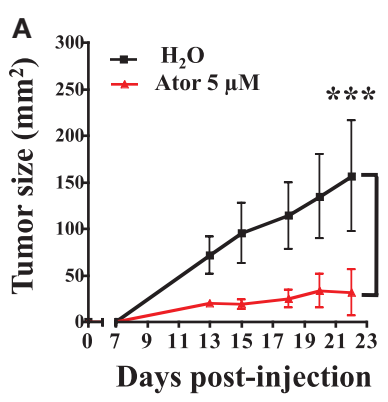

D

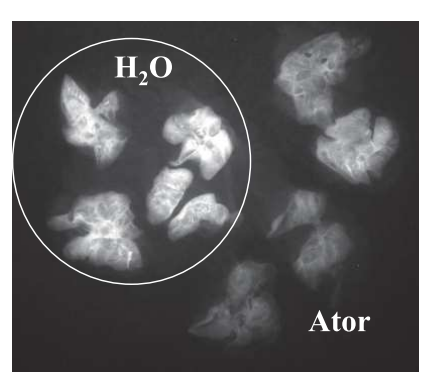

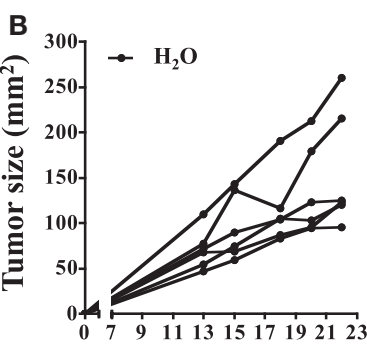

Days post-injection
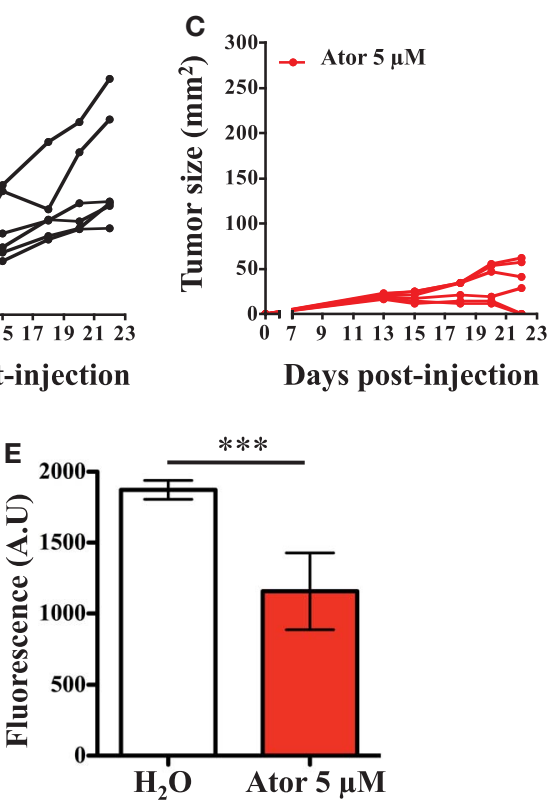

FIGURE 2 | Atorvastatin treatment inhibits melanoma in vivo development and metastasis. LB1319-MEL cells untreated $\left(\mathrm{H}_{2} \mathrm{O}\right)$ or pretreated with atorvastatin $5 \mu \mathrm{M}$ for $48 \mathrm{~h}$ (Ator) were injected subcutaneously into the flank of six nude mice. Tumor growth was measured every $2-3$ days (A). ${ }^{* *} P<0.001$; versus control condition using the two-way ANOVA test. Tumor growths for each mice are represented $(\mathbf{B}, \mathbf{C})$. LB1319-MEL cells untreated $\left(\mathrm{H}_{2} \mathrm{O}\right)$ or pretreated with
$5 \mu \mathrm{M}$ atorvastatin for $48 \mathrm{~h}$ (Ator) were injected intravenously. Fifteen days later IntegriSense was intravenously injected and the mice were sacrificed $30 \mathrm{~h}$ later. The lungs appeared fluorescent and the IntegriSense fluorescent signal was analyzed by Fluobeam®camera. The image is representative of one experiment (D). The fluorescent signal was quantified (E). ${ }^{* *} P<0.001$; versus control condition using the Student t-test. of MICA expression (Figure 3C), while cells transfected with RhoB- or RhoC-siRNAs significantly reduced MICA expression as compared to siCtrl (Figures 3D,E). Consequently, these Rho proteins cannot be responsible for the increased expression of MICA induced by statin treatment.

We then tested the impact of siRNA-induced reduction of Racl and Cdc42, two other essential members of the Rho family. These proteins are known to be involved in the regulation of several cellular functions, including migration and gene transcription (Heasman and Ridley, 2008). SiRNA transfections successfully inhibited Racl and Cdc42 expression (Figures A3D,E in Appendix). We observed that Cdc42 inhibition had no statistical effect on MICA expression, while Rac1 inhibition actually reduced MICA expression in LB1319-MEL cells (Figures 3F,G). Altogether, these results show that these Rho proteins are not responsible for the statin-induced overexpression of MICA.

Next, we investigated the role of Ras proteins in statin-induced MICA overexpression. Ras GTPase activities can be inhibited by statin treatment, because these proteins need to be farnesylated on their C-terminal end for membrane anchorage and activity. To determine whether Ras proteins are involved in MICA overexpression, we used a biochemical inhibitor of Ras protein activity ( $S$-FarneylThioSialicylic acid, FTS). The results showed that inhibition of Ras protein activity by FTS (50-100 $\mu \mathrm{M}$ for $24 \mathrm{~h}$ ) also induced a decrease in MICA expression (Figures 3H,I). Therefore, Ras proteins are not responsible for statin-induced MICA overexpression.

\section{PPAR $\gamma$ AND SAPK/JNK INVOLVEMENT IN STATIN-INDUCED MICA OVEREXPRESSION}

As it is known that statins may affect the PPAR $\gamma$ pathway (Balakumar and Mahadevan, 2012), we checked the involvement of this factor in the statin-induced MICA overexpression. As shown in Figures 4A,B, treatment of the LB1319-MEL cells with T0070907, a specific inhibitor of the PPAR $\gamma$ pathway, led to a weak but significant and reproducible decrease of the membrane MICA expression. Additionally, transfection of an expression vector encoding a dominant negative mutant of PPAR $\gamma(\operatorname{PPAR} \gamma \mathrm{DN})$ had a similar effect (Figures 4C,D). Furthermore, LB1319-MEL cells transfected with a reporter vector containing three PPAR $\gamma$ Responsive Element (PPRE) cloned upstream of a luciferase gene (pGL4.3xPPRE-FLuc) exhibited a weak but significant increase of the luciferase expression following $5 \mu \mathrm{M}$ atorvastatin treatment (Figure 4E). The treatment of LB1319-MEL cells with increasing doses of atorvastatin, from 0.3 to $10 \mu \mathrm{M}$, confirmed this data (Figure A4A in Appendix). Finally, CPT1A mRNAs were increased in LB1319-MEL cells following $5 \mu \mathrm{M}$ atorvastatin treatment (Figure A4B in Appendix). This data confirm the involvement of PPAR $\gamma$ pathway since CPT1A is a target gene for PPAR $\gamma$ (Heinaniemi et al., 2007; Szatmari et al., 2007; Sharma et al., 2012). Therefore, it is possible that statins activate the PPAR $\gamma$ pathway, leading to downstream increases in MICA expression.

To assess the possible involvement of other pathways, we then performed a Human Phospho-Kinase Array (R\&D systems). The 


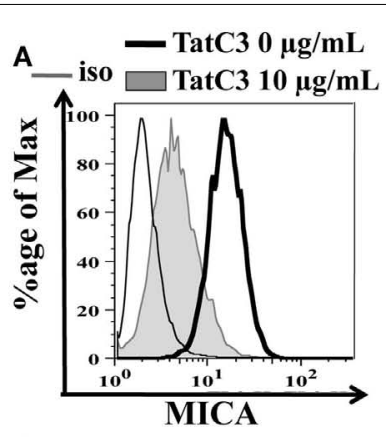

D

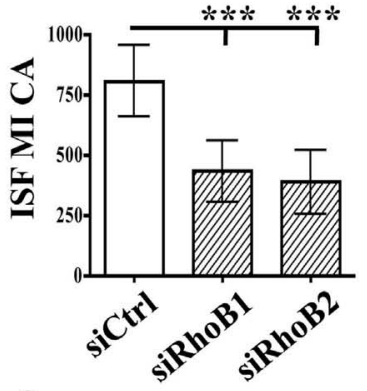

G

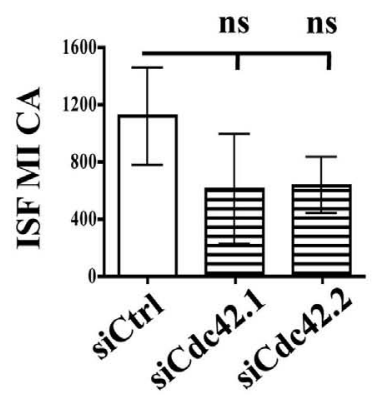

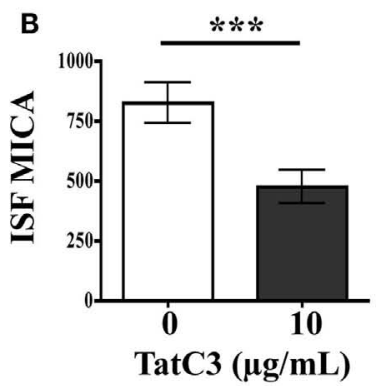

E

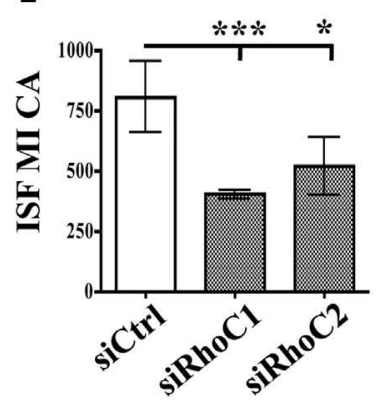

H

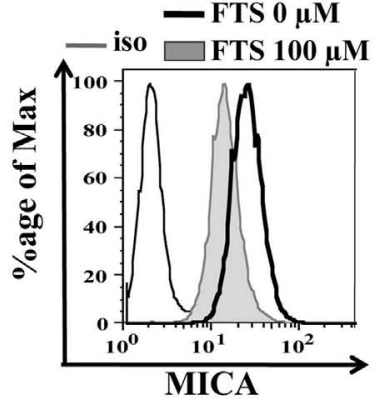

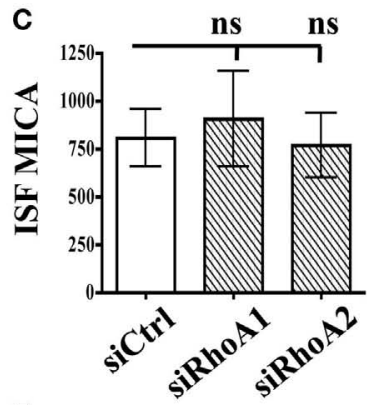

$\mathbf{F}$
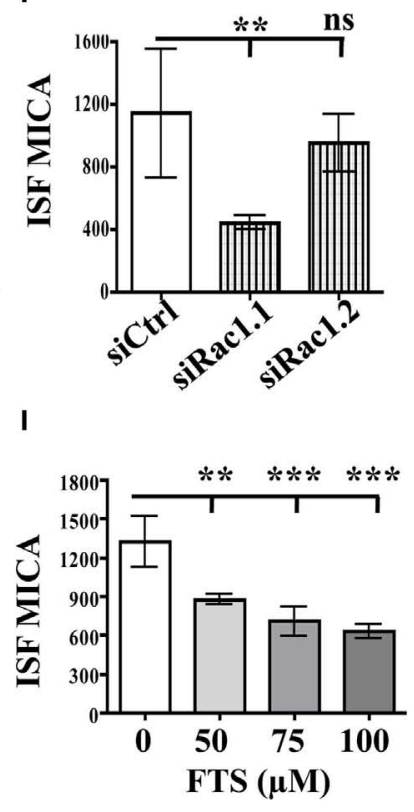

FIGURE 3 | Inhibition of Rho and Ras GTPases proteins do not induce MICA membrane overexpression. LB1319-MEL cells were untreated or treated with TatC3 exoenzyme (TatC3) $10 \mu \mathrm{g} / \mathrm{mL}$ for $24 \mathrm{~h}(\mathbf{A}, \mathbf{B})$.

LB1319-MEL cells were transfected with control siRNA (siCtrl) (C-G), two RhoA-specific siRNAs (siRhoA1, siRhoA2) (C), two RhoB-specific siRNAs (siRhoB1, siRhoB2) (D), two RhoC-specific siRNAs (siRhoC1, siRhoC2)

(E), two Rac1-specific siRNAs (siRac1.1, siRac1.2) (F), or two Cdc42-specific siRNAs (siCdc42.1, siCdc42.2) (G). LB1319-MEL cells were treated or not with a Ras inhibitor, FTS, 50-100 $\mu \mathrm{M}$ for $24 \mathrm{~h}(\mathbf{H}, \mathbf{I})$.
MICA membrane expression was analyzed by flow cytometry $72 \mathrm{~h}$ after transfection or $24 \mathrm{~h}$ after treatment. Representative illustrations of untreated cells (black) and of treated cells (filled in gray) with Tat-C3 exoenzyme (A) or FTS $\mathbf{( H )}$ are shown. To evaluate membrane antigen expression, the index of specific fluorescence (ISF) was calculated as indicated in the Section "Materials and Methods." Results are expressed as mean $\pm \mathrm{SD}$ (error bars, $n=3$ experiments). ${ }^{*} P<0.5$; ${ }^{*} P<0.01$; ** $P<0.001$ versus control condition using the Student $t$-test $(\mathbf{B})$ or the Tukey ANOVA test (C-I). obtained results, illustrated in Figures 4F,G, show that atorvastatin treatment enhanced phosphorylations of c-Jun N-terminal kinases (JKN, spot 2 in Figures 4F,G) and especially the c-Jun kinase on Serine 63 (c-Jun, spot 3 in Figures 4F,G). Atorvastatin could therefore increase MICA expression via the Stress Activated Protein Kinase/JNK (SAPK/JNK) pathway, as previously described in human kidney endothelial cells (Holmen et al., 2007).

Altogether, these results show that PPAR $\gamma$ and SAPK/JNK pathways are involved in the atorvastatin-induced MICA overexpression.

\section{DISCUSSION}

Melanomas are immunogenic tumors, which express tumor antigens and other molecules recognized by the effectors of innate and adaptive immune responses. The current metastatic melanoma immunotherapies have limited efficiency (Robert et al., 2011; Brahmer et al., 2012). Therefore, new molecules to enhance the immune response are still needed. Statins are well known for their hypocholesterolemic effect, but retrospective studies have shown that statins also have a protective effect against melanoma development (Jacobs et al., 2011). We previously showed that inhibitors of the mevalonate pathway, notably statins, favored an anti-melanoma adaptive immune response (Tilkin-Mariame et al., 2005; Sarrabayrouse et al., 2007, 2010). Here, we tested the ability of statins to enhance the NK-dependent innate immune response against human melanomas, particularly because these innate immune responses are essential for the control of metastases (Mehlen and Puisieux, 2006). 


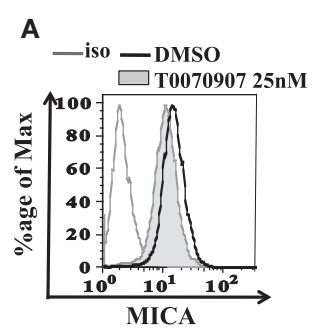

E

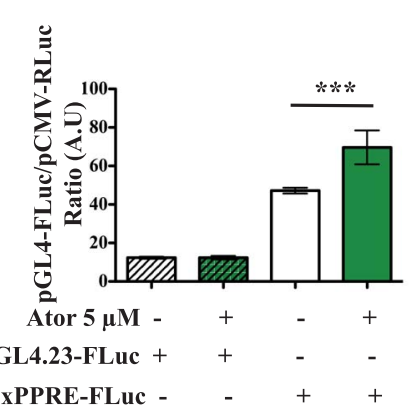

FIGURE 4 | PPAR $\gamma$ and SAPK/JNK are involved in atorvastatin-induced MICA overexpression. LB1319-MEL cells were treated with DMSO or a PPAR $\gamma$ inhibitor, T0070907 $25 \mathrm{nM}$ for $24 \mathrm{~h}$ (A,B). LB1319-MEL cells were transfected with an empty plasmid (Ctrl) or a plasmid encoding a PPAR $\gamma$ dominant negative mutant (PPAR $\gamma$ DN) (C,D). MICA membrane expression was analyzed $24 \mathrm{~h}$ after treatment or transfection. Representative illustrations of controlled cells (black) and treated cells (filled in gray) with T0070907 (A) or PPAR $\gamma$ DN (C) are shown. The ISF were calculated (B,D). LB1319-MEL cells were transfected with Firefly Luc control plasmids (pGL4.23-FLuc) or plasmid reporting the PPAR $\gamma$ PPRE activity (pGL4.3xPPRE-FLuc) and these cells were untreated $\left(\mathrm{H}_{2} \mathrm{O}\right)$ or treated
C

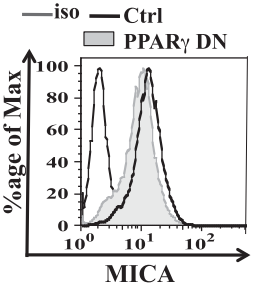

$\mathbf{F}$

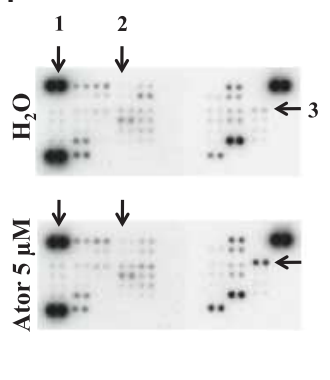

D

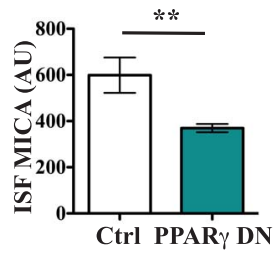

G

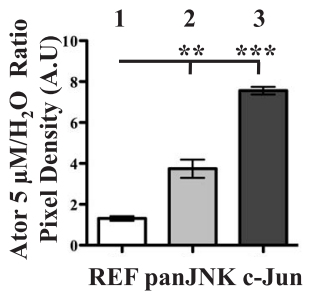

with atorvastatin $5 \mu \mathrm{M}$ (Ator $5 \mu \mathrm{M}$ ). Twenty-four hours later, luciferase assays were performed (E). LB1319-MEL cells were untreated $\left(\mathrm{H}_{2} \mathrm{O}\right)$ or treated with atorvastatin $5 \mu \mathrm{M}$ for $48 \mathrm{~h}$ and a Proteome Profiler Array was performed to compare kinases phosphorylation $(\mathbf{F}, \mathbf{G})$. One, two, and three correspond respectively to a reference spot (REF), panJNKT182/Y185, T221/Y223 (panJNK), and c-Jun S63 (c-Jun) phosphorylations (F). The densities of the signals were analyzed with ImageJ software and the ratios of atorvastatin on untreated conditions were calculated (G). Results are expressed as mean \pm SD (error bars, $n=3$ experiments). ${ }^{*} P<0.5$; ${ }^{*} P<0.01$; ${ }^{*}{ }^{*} P<0.001$ versus control condition using the Student $t$-test (B,D) or the Tukey ANOVA test $(\mathbf{E}, \mathbf{G})$.
In two human melanoma cell lines, we showed that statin treatments were not toxic at the doses used and that they weakly and reproducibly upregulated MICA expression, which is a critical target for anti-tumor NK cells. Interestingly, this weak MICA overexpression was able to enhance melanoma cell sensitivity to NK cell lysis in vitro. Similarly, Zhang et al. (2009) showed that sodium butyrate treatment of HeLa and HepG2 cells induced a weak but significant MICA overexpression allowing NK cells induceddeath enhancement. Furthermore as previously described using hydralazine and valproate treatment on epithelial cancer cell lines (Chavez-Blanco et al., 2011), our treatment of melanoma cells with statins induced MICA membrane overexpression without increasing the level of soluble MICA avoiding tumor-induced suppression of NK cytotoxicity against the tumor. Moreover, the efficacy of this statin-induced MICA overexpression was quite obvious in our in vivo experiments performed in nude mice. The innate immune response of nude mice could be tested against human melanoma cells because these mice have NK cells bearing NKG2D receptors, which are able to recognize human antigens, like MICA (Cerwenka and Lanier, 2001; Fuertes et al., 2008). In vivo experiments showed that melanoma tumor growth, induced by subcutaneous injections of statin-pretreated melanoma cells, was strongly decreased compared to the tumors induced by untreated melanoma cells. Furthermore two mice out of six completely rejected atorvastatin pretreated tumor cells. Most importantly, statin pretreatment also induced a significant decrease in melanoma metastatic lung implantation and a highly significant modification and decrease of the neoangiogenesis of these lungs. These modifications were detected by IntegriSense fluorescence, which recognize $\alpha v \beta 3$ integrins overexpressed in the lungs during cancer cells colonization (Kimura et al., 2009; Mery et al., 2011; He et al., 2012; Valdivia et al., 2012). In vitro tumor migration is not affected by atorvastatin treatment (Figures A2D,E in Appendix) therefore NK-killing of MICA overexpressing melanoma cells seem to be determinant for the reduction of melanoma development in vivo, as suggested by the differences of tumor growth between MICA negative (LB583-MEL and LB2033-MEL), MICA-low (untreated LB1319-MEL), and MICA-higher (atorvastatin-treated LB1319MEL) melanoma cells.

These in vivo experiments could not be reproduced with the BB74-MEL model because these cells did not form tumors in nude mice. Two hypotheses could explain these results. The first is that the in vitro proliferation rate of BB74-MEL cells (Figure A1D in Appendix) is too slow compared to LB1319-MEL cells (Figure 1E). As we followed the in vivo tumor growth of both cell lines for the same amount of time, the BB74-MEL cells may not have had enough time to form tumors. The second possible explanation is that BB74-MEL cells could be rejected at once by the NK cells 
of the nude mice, because BB74-MEL cells express more MICA than LB1319-MEL cells (Figure A4C in Appendix) and do not express MHC class I molecules, which inhibit NK cells activity (Figure A4D in Appendix).

In an effort to determine the mechanism of action of this statin-induced MICA overexpression, we came to the unexpected conclusion that it is not induced by members of the Ras superfamily. Experiments done with bacterial, biochemical inhibitors, or siRNAs specific for Ras and Rho GTPases could not phenocopy statin-induced MICA overexpression. However, two other statinaltered pathways (Holmen et al., 2007; Balakumar and Mahadevan, 2012) seem to be involved, namely PPAR $\gamma$ and SAPK/JNK. Inhibitors of PPAR $\gamma$ factor induced a weak downregulation of MICA expression, while treatment with atorvastatin increased the activity of a synthetic promoter containing PPREs. Similarly, atorvastatin treatment also resulted in increased phosphorylation of JNK and c-Jun. The respective roles of signaling pathways involving PPAR $\gamma$ and SAPK/JNK in melanoma development and in MICA expression are still unknown and should be analyzed in further studies.

In previous works, we showed that inhibitors of the Rho GTPases favored adaptive anti-melanoma immune response (Tilkin-Mariame et al., 2005; Sarrabayrouse et al., 2007, 2010). In the present paper, we demonstrate that statins have another beneficial role in modifying melanoma cells, which is to render them more sensitive to the NK-killing. These results reinforce the idea that statins could be effective drugs for an immuno-based therapy of melanoma, by favoring adaptive and innate responses as well as reducing the metastatic process.

\section{MATERIALS AND METHODS}

\section{TUMOR CELL LINES AND ANIMALS}

The human melanoma cell lines LB1319-MEL, BB74-MEL, LB2033-MEL, and LB583-MEL were kindly provided by Pr. T. Boon (Ludwig Institute for Cancer Research, Brussels). They were maintained in culture by serial passages in culture medium composed of RPMI 1640 medium (Lonza) supplemented with $10 \%$ FCS, $1 \mathrm{mM}$ glutamine, $1 \%$ penicillin-streptomycinamphotericinB (Lonza), and they were monthly tested to be mycoplasma-free. Six- to nine-week-old female NMRI nude mice were obtained from Elevages Janvier. The experiments in mice have been done in the appropriate conditions of husbandry, experimentation and care, controlled by the Ethic Comity of the Institut Claudius Regaud under the control of the Regional Comity of Midi-Pyrénées (France). Our protocols were validated and received the agreement number ICR-2009-0011.

\section{TREATMENT OF MELANOMA CELLS}

Melanoma cells were treated with a synthetic statin: atorvastatin (Pfizer) at different doses from 0.3 to $10 \mu \mathrm{M}$ for 24 or $48 \mathrm{~h}$, or a natural statin: lovastatin (Calbiochem) at 1,5 , or $10 \mu \mathrm{M}$ for $48 \mathrm{~h}$, or a RhoA, B, and C inhibitor: Tat-C3 exoenzyme (produced in our laboratory) (Sarrabayrouse et al., 2007) at $10 \mu \mathrm{g} / \mathrm{mL}$ for $24 \mathrm{~h}$, or with a Ras inhibitor: $S$-FarneylThioSialicylic acid (Interchim) at increasing concentrations from 50 to $100 \mu \mathrm{M}$ for $24 \mathrm{~h}$, or a PPAR $\gamma$ inhibitor: T0070907 (Calbiochem) at $25 \mathrm{nM}$ for $24 \mathrm{~h}$. Untreated cells correspond to cells treated with equivalent volume of $\mathrm{H}_{2} \mathrm{O}$ for atorvastatin, Ethanol (EtOH) for lovastatin, $\mathrm{NaCl}$ for Tat-C3 exoenzyme, DMSO for FTS and T0070907.

\section{TRANSFECTION OF siRNAs OR PLASMIDS}

Melanoma cells were transiently transfected with siRNAs as previously described (Sarrabayrouse et al., 2007). Briefly, $5 \times 10^{5}$ cells were transfected with $20 \mathrm{nM}$ of siRNAs using Oligofectamine (Invitrogen). The siRNA duplexes were purchased from Eurogentec: non-targeting siControl (siCtrl; GACGUGGGACUGAAGGGGU-TT), siRhoA1 (GAAGUCAAGCAUUUCUGUC-TT), siRhoA2 (GCAGGUAGAGUUGGCUUUG-TT), siRhoB1 (CUAUGUGGGCCGACAUUGAG-TT), siRhoB2 (CCGUCUUCGAGAACUAUGU-TT), siRhoC1 (UAAGAAGGACCUGAGGCAA-TT), siRhoC2 (GACUAUGAUCGACUGCGC-TT), siRac1.1 (CACCACUGUCCCAACACUC-TT), siRac1.2 (AAGGAGAUUGGUGCUGUAA-TT), siCdc42.1 (GAUAACUCACCACUGUCCA-TT), siCdc42.2 (GACUCCUUUCUUGCUUGUU-TT).

Melanoma cells were transiently transfected with a plasmid encoding PPAR $\gamma$ dominant negative proteins. Briefly, $5 \times 10^{5}$ cells were transfected with $500 \mathrm{ng}$ of plasmids with JetPrime (Invitrogen) according to the manufacturer's instructions.

\section{FLOW CYTOMETRY ANALYSES}

Melanoma cells $\left(1 \times 10^{6}\right)$ were stained with PE-conjugated antiMICA mAb and isotype control purchased from R\&D Systems. After $1 \mathrm{~h}$ incubation the stained cells were analyzed on a FACSCalibur (Becton Dickinson) and the results were analyzed with FlowJo software (Tree Star). To evaluate membrane antigen expression on several independent experiments, the index of specific fluorescence (ISF) was determined. This was calculated using the following formula:

(Median Fluorescence Intensity MFI with specific antibody - MFI with isotype control) $\times 100$

$$
\text { MFI with isotype control }
$$

\section{WESTERN BLOT ANALYSES}

Cells were lysed in lysis buffer $(20 \mathrm{mM}$ Tris pH 7.6, $150 \mathrm{mM}$ $\mathrm{NaCl}, 2 \mathrm{mM}$ EDTA, $0.1 \%$ SDS, $0.5 \%$ NP-40, $1 \%$ proteases, and phosphatases inhibitors cocktails) and protein extracts were prepared by the standard procedure and then separated (50-200 $\mu \mathrm{g}$ protein/lane) on SDS-PAGE gels. Proteins were blotted onto polyvinylidendifluoride membranes. The filters were incubated at $4^{\circ} \mathrm{C}$ overnight with primary antibodies against MICA (R\&D Systems). Actin was used as a loading control (Chemicon). The membranes (Hybond-p, Amersham Biosciences) were then incubated with HRP-labeled secondary antibody (Immunotech) for $1 \mathrm{~h}$ at room temperature (RT) and then detected by chemiluminescence detection kit (ECL, Pierce). Band intensities were quantified using ImageJ software (National Institute of Health).

\section{ELISA ASSAY}

ELISA assays were performed using MICA DuoSet ELISA (R\&D Systems). Briefly, capture antibodies were coated onto 96-wells plates overnight. Wells were washed and incubated with blocking reagent for $1 \mathrm{~h}$ at RT. One hundred microliters of the supernatants to be tested were added in triplicate for $2 \mathrm{~h}$ and detection antibody (in $2 \%$ goat serum; Lonza) was next incubated for $2 \mathrm{~h}$ at RT. 
One hundred microliters of Streptavidin-HRP were incubated for $20 \mathrm{~min}$ in the dark at RT and the reaction was revealed by TBM (Thermo-Scientific) after $20 \mathrm{~min}$ in the dark at RT. The reaction was stopped by addition of sulfuric acid $1 \mathrm{~N}$. Optical density was measured on a Labtech spectrophotometer at $450 \mathrm{~nm}$.

\section{NK CELLS ISOLATION}

NK cells isolations were performed using CD49b (DX5) MicroBeads (Miltenyi Biotec). Briefly, lymphocytes were isolated from three C57BL/6 spleens. Lymphocytes were then labeled with CD49b antibody coated with magnetic MicroBeads and applied onto a column placed on a magnetic support. The column was washed and unlabeled cells were collected. The CD49b-labeled NK cells were collected with PBS after the column removal from the magnetic support. NK cells purification was evaluated using FITC-conjugated CD49b antibody (>90\%).

\section{ANALYSIS OF NK CELLS CYTOTOXIC ACTIVITY}

We used Cytoxilux kit (Onco-immumin, Interchim) to investigate NK cells cytotoxic activity by flow cytometry. Briefly $1 \times 10^{6}$ LB1319-MEL cells pretreated either with $\mathrm{H}_{2} \mathrm{O}$ or $5 \mu \mathrm{M}$ atorvastatin for $48 \mathrm{~h}$ were stained with targets marker TFL-4 (FL-4) for $20 \mathrm{~min}$ at $37^{\circ} \mathrm{C}$ and then washed with PBS. Stained target cells were then diluted at $8 \times 10^{4}$ cells per $100 \mu \mathrm{L}$ of culture medium. The effector NK cells (E) were incubated with the stained target cells (T) for $1 \mathrm{~h}$ at $37^{\circ} \mathrm{C}$ at 5:1 E/T ratio in 96-well plates (Dutscher) in triplicate. Supernatant of each well was then discarded and $75 \mu \mathrm{L}$ of caspase substrate, which is FITC fluorescent when cleaved (FL1) or $75 \mu \mathrm{L}$ of Wash Buffer for control conditions were added. Cells were finally washed with Wash Buffer and resuspended in PBS for analysis on a FACSCalibur. The percentage of cytotoxicity was calculated as the percentage of dead target cells, stained both green $(\mathrm{FL}-1+)$ and blue $(\mathrm{FL}-4+)$, among the total number of target cells (all FL-4+ cells).

\section{SUBCUTANEOUS TUMOR GROWTH}

To study the tumor growth, NMRI nude mice were injected subcutaneously (s.c.) with $5 \times 10^{6}$ LB1319-MEL cells or $2 \times 10^{6}$ LB2033-MEL or LB583-MEL cells either untreated or pretreated with $5 \mu \mathrm{M}$ atorvastatin for $48 \mathrm{~h}$. The melanoma cells were washed thrice in PBS before the injections to completely eliminate the statin. Animals were monitored for tumor growth every 2-3 days by palpation and diameters of the tumors were measured using a Vernier caliper. Tumor-bearing animals were sacrificed at day 15 or 22 after tumor injection. At this time no mice were died and the tumors did not display severe ulceration or reached a size of $300 \mathrm{~mm}^{2}$. For the mice that had rejected the tumor cells, experiments were terminated 9 weeks after tumor implantation. Two groups of three mice were tested and the experiments were done twice. Results are expressed as surface $\pm S D$ (error bars, $n=6$ mice). Statistical analysis was performed using a two-way ANOVA test.

\section{PULMONARY METASTASES IMPLANTATION}

To study pulmonary metastases implantation, NMRI nude mice were injected i.v. with $1 \times 10^{6}$ LB1319-MEL cells either untreated or pretreated $48 \mathrm{~h}$ with $5 \mu \mathrm{M}$ atorvastatin. The melanoma cells were washed thrice in PBS before the injections to completely eliminate the statin. These i.v. injections in the tail vein of melanoma cells followed by lungs examination for tumor colonization allows controlled and quantitative experimentation of the last step of metastasis: the organ colonization. Mice were sacrificed 16 days later, at this time no mice were died. Mice were i.v. injected with $100 \mu \mathrm{L}$ of IntegriSense (Perkin-Elmer) $30 \mathrm{~h}$ before sacrifice. The fluorescence in the lungs was analyzed by Fluobeam ${ }^{\circledR} \mathrm{cam}$ era $(690-700 \mathrm{~nm})$ for $120 \mathrm{~ms}$ exposition and the fluorescent signals were quantified using ImageJ software (National Institute of Health). The experiments included three or four mice/group and were done twice.

\section{IN VITRO ASSAYS FOR CELL MIGRATION}

The migration assay was performed with $8-\mu \mathrm{m}$ pore size transwell system (BD Biosciences). Cells $\left(1 \times 10^{4} /\right.$ well $)$ were added with serum-free medium in the upper compartment of the filter. The bottom chamber was filled with complete medium. After $24 \mathrm{~h}$, cells on the bottom surface of the filter were stained and counted under an Eclipse Ti microscope (Nikon Instruments; objective $20 \times$ ) and a CoolSNAP HQ2 camera (Photometrics) in three randomized fields of $505 \mathrm{~mm}^{2}$.

\section{LUCIFERASE ASSAYS}

The reporter vector for PPAR $\gamma$, pGL4.3xPPRE-FLuc plasmid containing PPRE sequences, was homemade constructed from the pB3xPPRE-Luc in an empty vector pGL4.23-FLuc (Rauwel et al., 2010) expressing Firefly Luciferase (FLuc). The pGL4.3xPPRE and the pGL4.23 were transfected using JetPrime (Invitrogen). The pCMV-RLuc plasmid expressing Renilla Luciferase (RLuc) was co-transfected as an internal control. Briefly, $3 \times 10^{4}$ cells were transfected with the reporter vectors as described above. Four hours after, transfection was stopped by removing the medium and cells were treated with $\mathrm{H}_{2} \mathrm{O}$ or atorvastatin. Twenty-four hours later, luciferase activities were measured using the Dual Luciferase Assay System (Promega).

\section{REAL-TIME QUANTITATIVE PCR}

Total RNA of LB1319-MEL cells untreated and treated with atorvastatin 5 or $10 \mu \mathrm{M}$ for $24 \mathrm{~h}$ were isolated using the RNeasy mini kit (Qiagen) combined with the RNase-Free DNase set (Qiagen) according to the manufacturer's instructions, then reversetranscribed using the iScript cDNA synthesis kit (BioRad). Quantitative real-time PCR was performed with a CFX96 Touch RealTime PCR detection system (BioRad) using iQ SYBR Green Supermix (BioRad). We used $\beta$-actin as a housekeeping gene. Primers sequences were: CPT1A forward 5'TCG-ATT-TTC-AAGGGT-CTT-CG3', CPT1A reverse 5'CAC-AAC-GAT-CAG-CAAACT-GG3', $\beta$-actin forward 5'TCC-CTG-GAG-AAG-AGC-TACGA3 ${ }^{\prime}, \beta$-actin reverse $5^{\prime}$-AAG-GAA-GGC-TGG-AAG-AG3'. The relative fold change was calculated using the following formula:

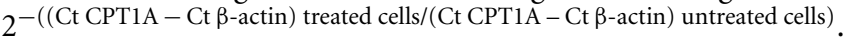

\section{PROTEOME PROFILER ARRAY}

Proteome Profiler Arrays were performed using Human PhosphoKinase Array Kit (R\&D Systems). Briefly, $5 \times 10^{5}$ LB1319-MEL cells, either untreated $\left(\mathrm{H}_{2} \mathrm{O}\right)$ or treated with $5 \mu \mathrm{M}$ atorvastatin, were lysed in the Lysis buffer. Supernatants were incubated on the blocked membranes overnight at $4^{\circ} \mathrm{C}$. Membranes 
were washed and incubated with detection antibodies cocktails for $2 \mathrm{~h}$ at RT. Membranes were washed and incubated with $1 \mathrm{~mL}$ of diluted Streptavidin-HRP for $30 \mathrm{~min}$ in the dark at RT. Then the phospho-kinases were detected using chemiluminescence detection. Spot intensities were quantified using ImageJ software (National Institute of Health).

\section{REFERENCES}

Balakumar, P., and Mahadevan, N. (2012). Interplay between statins and PPARs in improving cardiovascular outcomes: a doubleedged sword? Br. J. Pharmacol. 165, 373-379.

Berman, D., Parker, S. M., Siegel, J., Chasalow, S. D., Weber, J., Galbraith, S., et al. (2010). Blockade of cytotoxic T-lymphocyte antigen-4 by ipilimumab results in dysregulation of gastrointestinal immunity in patients with advanced melanoma. Cancer Immun. 10, 11.

Boudreau, D. M., Yu, O., and Johnson, J. (2010). Statin use and cancer risk: a comprehensive review. Expert Opin. Drug. Saf. 9, 603-621.

Brahmer, J. R., Tykodi, S. S., Chow, L. Q., Hwu, W. J., Topalian, S. L., Hwu, P., et al. (2012). Safety and activity of anti-PD-L1 antibody in patients with advanced cancer. N. Engl. J. Med. 366, 2455-2465.

Cerwenka, A., and Lanier, L. L. (2001). Ligands for natural killer cell receptors: redundancy or specificity. Immunol. Rev. 181, 158-169.

Chavez-Blanco, A., De la CruzHernandez, E., Dominguez, G. I., Rodriguez-Cortez, O., Alatorre, B., Perez-Cardenas, E., et al. (2011). Upregulation of NKG2D ligands and enhanced natural killer cell cytotoxicity by hydralazine and valproate. Int. J. Oncol. 39, 1491-1499.

Flaherty, K. T., Puzanov, I., Kim, K. B., Ribas, A., McArthur, G. A., Sosman, J. A., et al. (2010). Inhibition of mutated, activated BRAF in metastatic melanoma. N. Engl. J. Med. 363, 809-819.

Fuertes, M. B., Girart, M. V., Molinero, L. L., Domaica, C. I., Rossi, L. E., Barrio, M. M., et al. (2008). Intracellular retention of the NKG2D ligand MHC class I chain-related gene $\mathrm{A}$ in human melanomas confers immune privilege and prevents NK cell-mediated cytotoxicity. J. Immunol. 180, 4606-4614.

Guerra, N., Tan, Y. X., Joncker, N. T., Choy, A., Gallardo, F., Xiong, N., et al. (2008). NKG2D-deficient mice are defective in tumor surveillance in models of spontaneous malignancy. Immunity 28, 571-580.

He, T., Xue, Z., Lu, K. M., Valdivia, Y. A. M., Wong, K. K., Xie, W., et al. (2012). A minimally invasive multimodality image-guided (MIMIG) system for peripheral lung cancer intervention and diagnosis. Comput. Med. Imaging Graph 36, 345-355.

Heasman, S. J., and Ridley, A. J. (2008). Mammalian Rho GTPases: new insights into their functions from in vivo studies. Nat. Rev. Mol. Cell Biol. 9, 690-701.

Heinaniemi, M., Uski, J. O., Degenhardt, T., and Carlberg, C. (2007). Metaanalysis of primary target genes of peroxisome proliferator-activated receptors. Genome Biol. 8, R147.

Hodi, F. S., O’Day, S. J., McDermott, D. F., Weber, R.W., Sosman, J. A., Haanen, J. B., et al. (2010). Improved survival with ipilimumab in patients with metastatic melanoma. N. Engl. J. Med. 363, 711-723.

Holmen, C., Elsheikh, E., Christensson, M., Liu, J., Johansson, A. S., Qureshi, A. R., et al. (2007). Anti endothelial cell autoantibodies selectively activate SAPK/JNK signalling in Wegener's granulomatosis. J. Am. Soc. Nephrol. 18, 2497-2508.

Jacobs, E. J., Newton, C. C., Thun, M. J., and Gapstur, S. M. (2011). Longterm use of cholesterol-lowering drugs and cancer incidence in a large United States cohort. Cancer Res. 71, 1763-1771.

Kimura, R. H., Cheng, Z., Gambhir, S. S., and Cochran, J. R. (2009). Engineered knottin peptides: a new class of agents for imaging integrin expression in living subjects. Cancer Res. 69, 2435-2442.

Lanier, L. L. (2008). Up on the tightrope: natural killer cell activation and inhibition. Nat. Immunol. 9, 495-502.

Mehlen, P., and Puisieux, A. (2006). Metastasis: a question of life or death. Nat. Rev. Cancer 6, 449-458.

Mery, E., Jouve, E., Guillermet, S., Bourgognon, M., Castells, M., Golzio, M., et al. (2011). Intraoperative fluorescence imaging of peritoneal dissemination of ovarian carcinomas. A preclinical study. Gynecol. Oncol. 122, 155-162.

Meyer, S., Vogt, T., Landthaler, M., Berand, A., Reichle, A., Bataille, F., et al. (2009). Cyclooxygenase 2 (COX2) and peroxisome proliferator-activated receptor

\section{ACKNOWLEDGMENTS}

The authors thank Pr. T. Boon for providing the human melanoma cell lines and Dr. S. Chavanas for the dominant negative mutant and the inhibitor of PPARg. This work was supported by the Institut National de la Santé et de la Recherche Médicale INSERM and by the Institut Claudius Regaud ICR.

gamma (PPARG) are stagedependent prognostic markers of malignant melanoma. PPAR Res. 2009, 848645.

Peters, J. M., Shah, Y. M., and Gonzalez, F. J. (2012). The role of peroxisome proliferator-activated receptors in carcinogenesis and chemoprevention. Nat. Rev. Cancer 12, 181-195.

Rauwel, B., Mariame, B., Martin, H., Nielsen, R., Allart, S., Pipy, B., et al. (2010). Activation of peroxisome proliferator-activated receptor gamma by human cytomegalovirus for de novo replication impairs migration and invasiveness of cytotrophoblasts from early placentas. J. Virol. 84 2946-2954.

Rikitake, Y., and Hirata, K. (2009). Inhibition of RhoA or Racl? Mechanism of cholesterol-independent beneficial effects of statins. Circ. J. 73, 231-232.

Robert, C., Thomas, L., Bondarenko, I., O'Day, S., Weber, J., Garbe, C., et al. (2011). Ipilimumab plus dacarbazine for previously untreated metastatic melanoma. N. Engl. J. Med. 364, 2517-2526.

Sarrabayrouse, G., Pich, C., Moriez, R., Armand-Labit, V., Rochaix, P., Favre, G., et al. (2010). Melanoma cells treated with GGTI and IFN-gamma allow murine vaccination and enhance cytotoxic response against human melanoma cells. PLoS ONE 5:e9043. doi:10.1371/journal.pone.0009043

Sarrabayrouse, G., Synaeve, C., Leveque, K., Favre, G., and TilkinMariame, A. F. (2007). Statins stimulate in vitro membrane FasL expression and lymphocyte apoptosis through RhoA/ROCK pathway in murine melanoma cells. Neoplasia 9, 1078-1090.

Sharma, S., Sun, X., Rafikov, R., Kumar, S., Hou, Y., Oishi, P. E., et al. (2012). PPAR-gamma regulates carnitine homeostasis and mitochondrial function in a lamb model of increased pulmonary blood flow. PLoS ONE 7:e41555. doi:10.1371/journal.pone.0041555

Szatmari, I., Torocsik, D., Agostini, M., Nagy, T., Gurnell, M., Barta, E., et al. (2007). PPARgamma regulates the function of human dendritic cells primarily by altering lipid metabolism. Blood 110, 3271-3280.

Tilkin-Mariame, A. F., Cormary, C., Ferro, N., Sarrabayrouse, G., LajoieMazenc, I., Faye, J. C., et al. (2005). Geranylgeranyl transferase inhibition stimulates anti-melanoma immune response through $\mathrm{MHC}$ Class I and costimulatory molecule expression. FASEB J. 19, 1513-1515.

Valdivia, Y. A. M., Wong, K., He, T. C., Xue, Z., and Wong, S. T. (2012). Image-guided fiberoptic molecular imaging in a VX2 rabbit lung tumor model. J. Vasc. Interv. Radiol. 22, 1758-1764.

Weeraratna, A. T. (2012). RAF around the edges - the paradox of BRAF inhibitors. N. Engl. J. Med. 366, 271-273.

Zhang, C., Wang, Y., Zhou, Z., Zhang, J., and Tian, Z. (2009). Sodium butyrate upregulates expression of NKG2D ligand MICA/B in HeLa and HepG2 cell lines and increases their susceptibility to NK lysis. Cancer Immunol. Immunother. 58, 1275-1285.

Conflict of Interest Statement: The authors declare that the research was conducted in the absence of any commercial or financial relationships that could be construed as a potential conflict of interest.

Received: 18 December 2012; accepted: 27 February 2013; published online: 13 March 2013.

Citation: Pich C, Teiti I, Rochaix P, Mariamé B, Couderc B, Favre $G$ and Tilkin-Mariamé A-F (2013) Statins reduce melanoma development and metastasis through MICA overexpression. Front. Immunol. 4:62. doi:10.3389/fimmu.2013.00062

This article was submitted to Frontiers in Tumor Immunity, a specialty of Frontiers in Immunology.

Copyright (C) 2013 Pich, Teiti, Rochaix, Mariamé, Couderc, Favre and TilkinMariamé. This is an open-access article distributed under the terms of the Creative Commons Attribution License, which permits use, distribution and reproduction in other forums, provided the original authors and source are credited and subject to any copyright notices concerning any third-party graphics etc. 

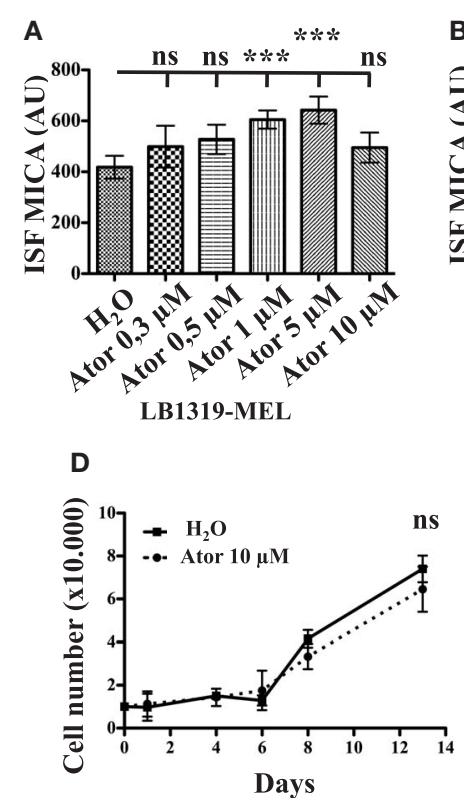

BB74-MEL

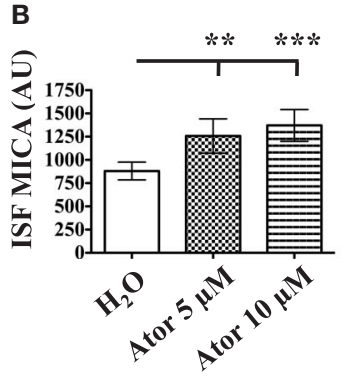

BB74-MEL

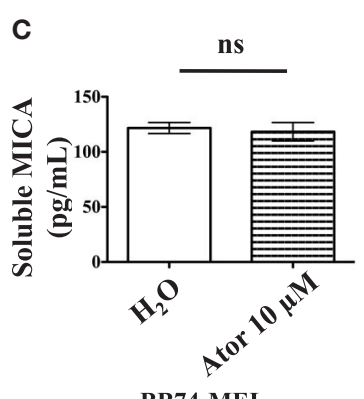

BB74-MEL

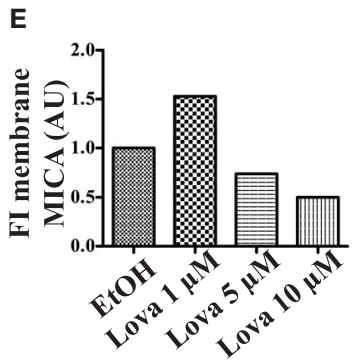

LB1319-MEL

FIGURE A1 | Atorvastatin and lovastatin treatments of LB1319-MEL and BB74-MEL cells induce MICA membrane overexpression. LB1319-MEL cells were treated with increasing concentrations of atorvastatin and membrane MICA expression was analyzed by flow cytometry (A). BB74-MEL cells were treated with 5 or $10 \mu \mathrm{M}$ atorvastatin for $48 \mathrm{~h}$ (Ator) or untreated $\left(\mathrm{H}_{2} \mathrm{O}\right)$. MICA membrane expression was analyzed by flow cytometry $(\mathbf{B})$ and cleavage by ELISA (C). ${ }^{*} P<0.01 ;{ }^{*}{ }^{*} P<0.001$; versus control condition using the Tukey ANOVA test. Every 2-3 days pretreated (Ator) and untreated $\left(\mathrm{H}_{2} \mathrm{O}\right)$ BB74-MEL cells were counted to evaluate the in vitro proliferation (D). ns; versus control condition using the two-way ANOVA test. LB1319-MEL cells were treated with increasing concentrations of lovastatin and membrane MICA expression was analyzed (E).

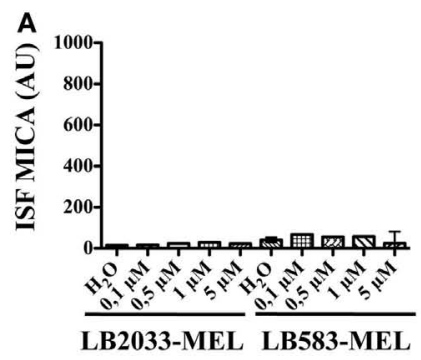

D

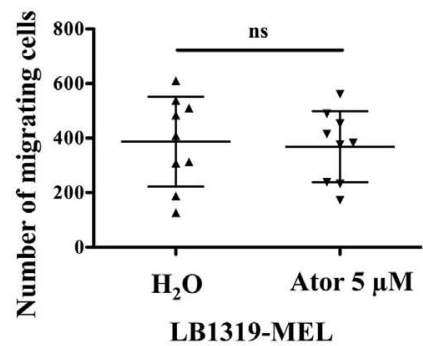

FIGURE A2 | Atorvastatin treatment had no effect on MICA negative melanoma cell lines in vivo tumor growth and on in vitro tumor migration. LB2033-MEL and LB583-MEL cells were treated with increasing concentrations of atorvastatin for $48 \mathrm{~h}$ and MICA membrane expression was analyzed by flow cytometry (A). ns; versus control condition using the Tukey ANOVA test. LB2033-MEL and LB583-MEL cells untreated $\left(\mathrm{H}_{2} \mathrm{O}\right)$ or pretreated with atorvastatin $5 \mu \mathrm{M}$

E
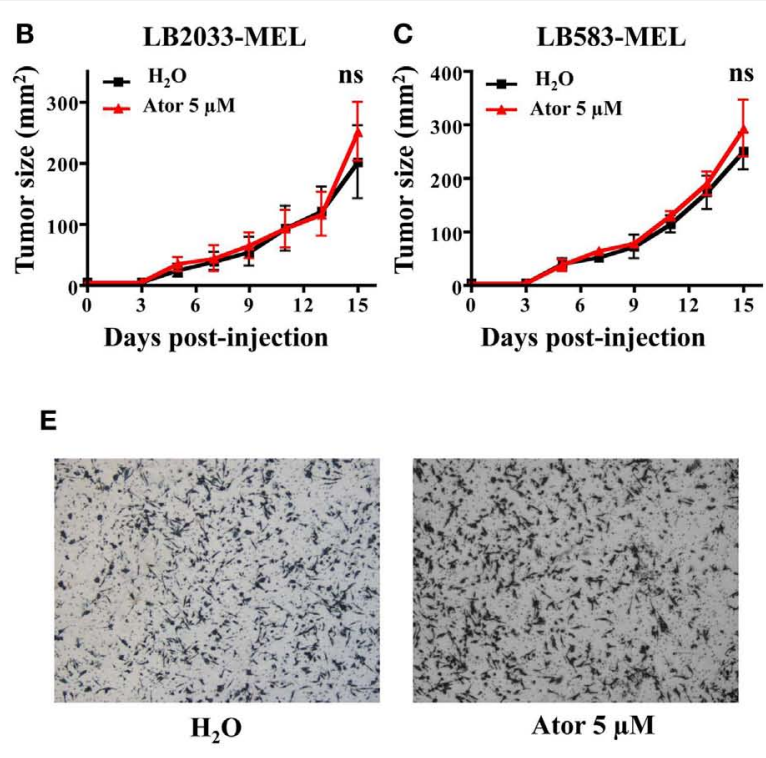

Ator $5 \mu \mathrm{M}$

LB1319-MEL

for $48 \mathrm{~h}$ (Ator) were injected subcutaneously into the flank of nude mice. Tumor growth was measured every 2-3 days (B,C). ns; versus control condition using the two-way ANOVA test. In vitro migration of LB1319-MEL cells untreated $\left(\mathrm{H}_{2} \mathrm{O}\right)$ or pretreated with atorvastatin $5 \mu \mathrm{M}$ for $48 \mathrm{~h}$ (Ator) was analyzed using transwell assays (D). ns; versus control condition using the Student $t$-test. Images are representative of the migrating cells (E). 

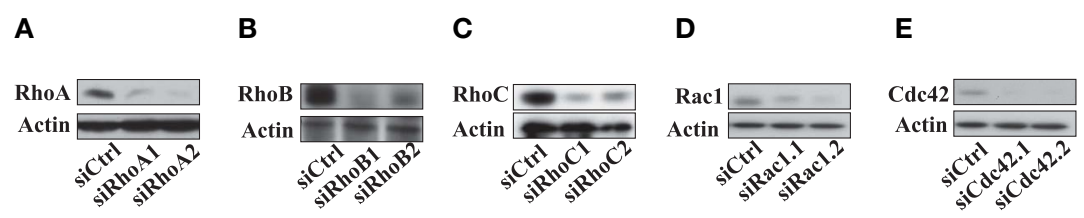

FIGURE A3 | Decrease of Rho proteins expression in LB1319-MEL cells transfected with Rho specific siRNAs. The efficiency in decreasing Rho

western blot, and actin was used as a control of a uniform loading. RhoA, protein expression of Rho specific siRNAs transfections were analyzed by

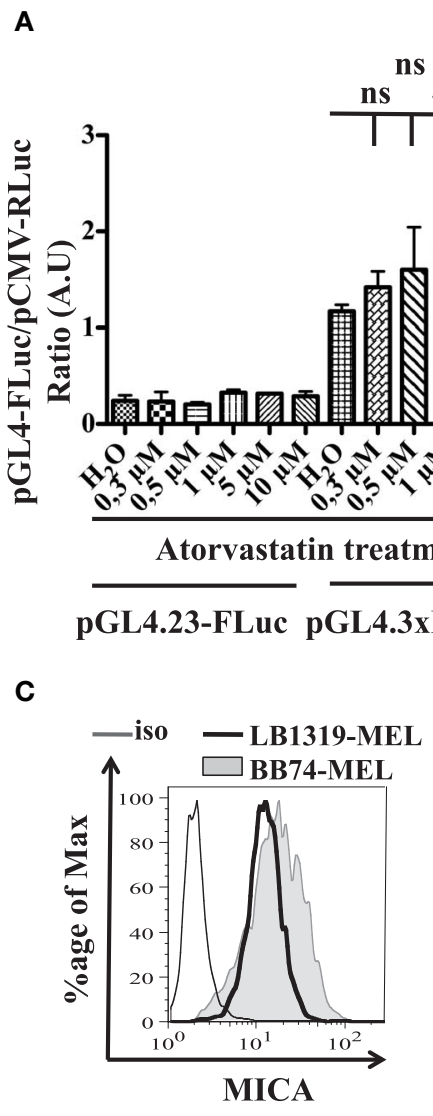

FIGURE A4 | Activation of PPAR $\gamma$ pathway by increasing doses of atorvastatin and MICA and MHC class I expression in LB1319-MEL versus BB47-MEL cell lines. LB1319-MEL cells were transfected with Firefly Luc control plasmids (pGL4.23-FLuc) or plasmid reporting the PPAR $\gamma$ PPRE activity (pGL4.3xPPRE-FLuc) and these cells were untreated $\left(\mathrm{H}_{2} \mathrm{O}\right)$ or treated with increasing doses of atorvastatin from 0.3 to $10 \mu \mathrm{M}$. Twenty-four hours later, luciferase assays were performed (A). LB1319-MEL cells were

\section{B}

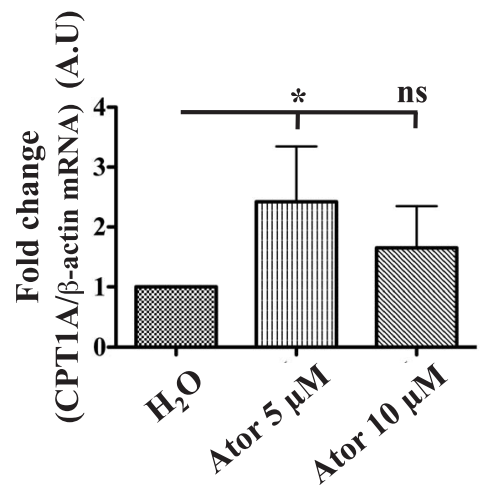

D

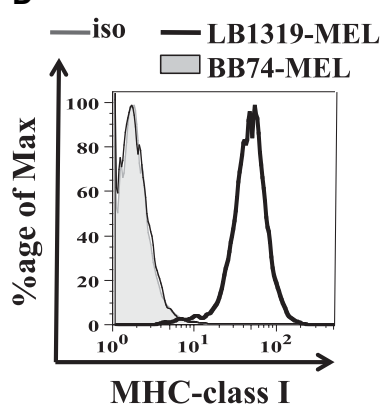

treated with 5 or $10 \mu \mathrm{M}$ atorvastatin for $24 \mathrm{~h}$ or untreated $\left(\mathrm{H}_{2} \mathrm{O}\right)$ and CPT1A mRNA relative expression, normalized by $\beta$-actin, was detected by RT-qPCR (B). Results are expressed as mean \pm SD (error bars, $n=3$ experiments). ${ }^{*} P<0.5$; ${ }^{*} P<0.01 ;{ }^{*}{ }^{*} P<0.001$ versus control condition using the Tukey ANOVA test (A,B). Expressions of MICA (C), and MHC class I molecules (D) were compared between LB1319-MEL and BB74-MEL cell lines by flow cytometry. 